\title{
On the global version of Euler-Lagrange equations
}

\author{
R E Gamboa Saraví ${ }^{1}$ and J E Solomin ${ }^{2}$ \\ ${ }^{1}$ Departamento de Física, Facultad de Ciencias Exactas, Universidad Nacional de La Plata, \\ Argentina \\ ${ }^{2}$ Departamento de Matemática, Facultad de Ciencias Exactas, Universidad Nacional de La Plata, \\ Argentina \\ E-mail: quique@fisica.unlp.edu.ar
}

Received 11 December 2002, in final form 21 May 2003

Published 18 June 2003

Online at stacks.iop.org/JPhysA/36/7301

\begin{abstract}
The introduction of a covariant derivative on the velocity phase space is needed for a global expression of Euler-Lagrange equations. The aim of this paper is to show how its torsion tensor turns out to be involved in such a version.
\end{abstract}

PACS numbers: $45.10 . \mathrm{Na}, 02.40 . \mathrm{Yy}$

An increasing attention has been recently paid to coordinate-free formulations of motion equations in classical mechanics (see for instance [1,2] and references therein). In this work we write down intrinsic Euler-Lagrange equations and show the appearance of a torsion term. Furthermore, we shall see that this term should also be present in the horizontal LagrangePoincare equations considered in [1,2], if the torsion of the chosen derivative does not vanish.

It is worth noting that covariant derivatives with non-vanishing torsion naturally arise in several branches of physics; namely dynamics with nonholonomic constraints [3, 4], E Cartan's theory of gravity (see for instance [5]) and modern string theories (see for example [6]), among others.

Let us consider a physical system with configuration manifold $Q$ and Lagrangian $L(q, \dot{q}): T Q \rightarrow \mathbb{R}$ (for this geometrical setting, see for instance [7]).

If a coordinate-free characterization of the Euler-Lagrange equations associated with the system is required a covariant derivative $\mathrm{D}$ must be introduced to $T Q$, for $\frac{\partial L}{\partial q}$ is involved (see for instance [8]). Once such $\mathrm{D}$ is chosen, $\frac{\mathrm{D} L}{\mathrm{D} q}$ is defined in the standard way

$$
\frac{\mathrm{D} L}{\mathrm{D} q}\left(q_{0}, \dot{q}_{0}\right)=\left.\frac{\partial}{\partial \lambda}\right|_{\lambda=0} L \circ \gamma(\lambda)
$$

with $\gamma(\lambda)=\left(q(\lambda), \dot{q}_{0 \|}(\lambda)\right), q(0)=q_{0}, \dot{q}(0)=\dot{q}_{0}$ and $\dot{q}_{0 \|}(\lambda)$ the parallel transport of $\dot{q}_{0}$ along $q(\lambda)$. 
Moreover, an associated covariant derivative on $T^{*} Q$, that we will also denote by $\mathrm{D}$, is naturally defined through the Leibnitz rule: for any curves $\alpha(t)$ and $v(t)$ in $T^{*} Q$ and $T Q$, respectively

$$
\frac{\mathrm{d}}{\mathrm{d} t}\langle\alpha(t), v(t)\rangle=\left\langle\frac{\mathrm{D} \alpha(t)}{\mathrm{D} t}, v(t)\right\rangle+\left\langle\alpha(t), \frac{D v(t)}{\mathrm{D} t}\right\rangle
$$

where $\langle$,$\rangle denotes the pairing between T^{*} Q$ and $T Q$.

It is worth noting that $\frac{\partial}{\partial \dot{q}}$ has a coordinate-free sense: it is the derivative along the fibre.

Proposition 1. Let $D$ be an arbitrary covariant derivative on $T Q$. Then the coordinate-free expression of the Euler-Lagrange equations is

$$
\frac{\mathrm{D}}{\mathrm{D} t}\left(\frac{\partial L}{\partial \dot{q}}\right)-\frac{\mathrm{D} L}{\mathrm{D} q}=\frac{\partial L}{\partial \dot{q}} T(\dot{q}(t),)
$$

where $T($, , ) is the torsion tensor of $D$.

Proof. The curve $q(t)$ is a solution of the Euler-Lagrange equations if and only if it is a critical point for the action

$$
S=\int_{t_{0}}^{t_{1}} L(q(t), \dot{q}(t)) \mathrm{d} t
$$

for variations of the curves such that $q_{0}$ and $q_{1}$ remain fixed. That is, for each $q(t, \lambda)$ : $\left[t_{0}, t_{1}\right] \times(-\varepsilon, \varepsilon) \rightarrow Q$ such that $q(t, 0)=q(t), q\left(t_{0}, \lambda\right)=q\left(t_{0}\right)$ and $q\left(t_{1}, \lambda\right)=q\left(t_{1}\right)$,

$$
\left.\frac{\partial}{\partial \lambda}\right|_{\lambda=0} \int_{t_{0}}^{t_{1}} L(q(t, \lambda), \dot{q}(t, \lambda)) \mathrm{d} t=\int_{t_{0}}^{t_{1}} \delta L(q(t), \dot{q}(t)) \mathrm{d} t=0
$$

where $\delta L(q(t), \dot{q}(t))=\left.\frac{\partial}{\partial \lambda}\right|_{\lambda=0} L(q(t, \lambda), \dot{q}(t, \lambda))$.

But

$$
\begin{aligned}
\delta L(q(t), \dot{q}(t))= & \lim _{\lambda \rightarrow 0} \frac{L(q(t, \lambda), \dot{q}(t, \lambda))-L(q(t, 0), \dot{q}(t, 0))}{\lambda} \\
= & \lim _{\lambda \rightarrow 0} \frac{L(q(t, \lambda), \dot{q}(t, \lambda))-L\left(q(t, \lambda), \dot{q}_{\|}(t, \lambda)\right)}{\lambda} \\
& +\lim _{\lambda \rightarrow 0} \frac{L\left(q(t, \lambda), \dot{q}_{\|}(t, \lambda)\right)-L(q(t, 0), \dot{q}(t, 0))}{\lambda}
\end{aligned}
$$

where $\dot{q}_{\|}(t, \lambda)$ is the parallel translate of the vector $\dot{q}(t, 0)$ along the curve $\left.q(t, \lambda)\right|_{\text {fixed } t}$ (see figure 1).

Then

$$
\delta L(q(t), \dot{q}(t))=\frac{\partial L}{\partial \dot{q}} \mathrm{D}_{\delta q(t)} \dot{q}(t)+\frac{\mathrm{D} L}{\mathrm{D} q} \delta q(t)
$$

where we have denoted

$$
\delta q(t)=\left.\frac{\partial q(t, \lambda)}{\partial \lambda}\right|_{\lambda=0} \quad \text { and } \quad \dot{q}(t)=\left.\frac{\partial q(t, \lambda)}{\partial t}\right|_{\lambda=0} .
$$

By definition of the torsion tensor $T($, ), we have

$$
T(\dot{q}(t), \delta q(t))=\mathrm{D}_{\dot{q}(t)} \delta q(t)-\mathrm{D}_{\delta q(t)} \dot{q}(t)-[\dot{q}(t), \delta q(t)] .
$$

Thus, by using (2) and taking into account that $[\dot{q}(t), \delta q(t)]$ vanishes, we have

$$
\begin{aligned}
\delta L(q(t), \dot{q}(t)) & =\frac{\partial L}{\partial \dot{q}} \mathrm{D}_{\dot{q}(t)} \delta q(t)+\frac{\mathrm{D} L}{\mathrm{D} q} \delta q(t)-\frac{\partial L}{\partial \dot{q}} T(\dot{q}(t), \delta q(t)) \\
& =\frac{\mathrm{d}}{\mathrm{d} t}\left(\frac{\partial L}{\partial \dot{q}} \delta q(t)\right)-\frac{\mathrm{D}}{\mathrm{D} t}\left(\frac{\partial L}{\partial \dot{q}}\right) \delta q(t)+\frac{\mathrm{D} L}{\mathrm{D} q} \delta q(t)-\frac{\partial L}{\partial \dot{q}} T(\dot{q}(t), \delta q(t)) .
\end{aligned}
$$




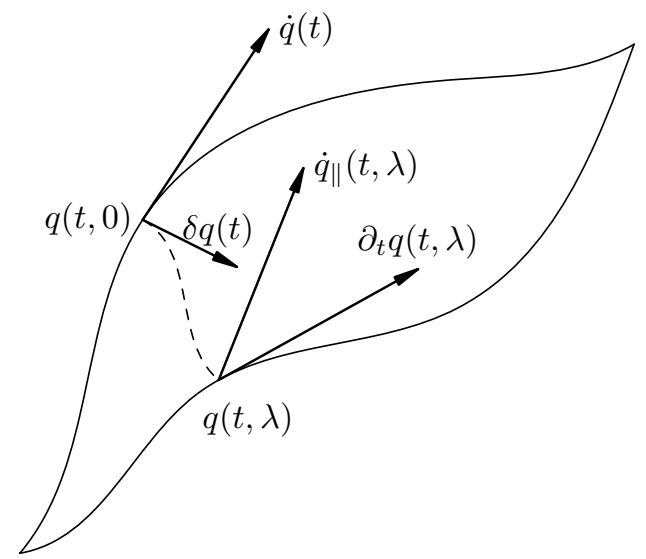

Figure 1. The vector $\dot{q}_{\|}(t, \lambda)$ is the parallel transport of the vector $\dot{q}(t, 0)$ along the dashed curve.

Now integrating along the curve $q(t)$ we finally get

$$
\frac{\mathrm{D}}{\mathrm{D} t}\left(\frac{\partial L}{\partial \dot{q}}\right) \delta q(t)-\frac{\mathrm{D} L}{\mathrm{D} q} \delta q(t)=\frac{\partial L}{\partial \dot{q}} T(\dot{q}(t), \delta q(t)) .
$$

Remark 1. Of course, regardless of the covariant derivative $\mathrm{D}$ we introduced, in any coordinate patch the Euler-Lagrange equations always read

$$
\frac{\mathrm{d}}{\mathrm{d} t}\left(\frac{\partial L}{\partial \dot{q}^{i}}\right)-\frac{\partial L}{\partial q^{i}}=0
$$

Remark 2. As well known (e.g., [8] ), a torsion-free D can always be chosen. It is obvious that for such a connection, global Euler-Lagrange equations read

$$
\frac{\mathrm{D}}{\mathrm{D} t}\left(\frac{\partial L}{\partial \dot{q}}\right)-\frac{\mathrm{D} L}{\mathrm{D} q}=0 .
$$

So in this case, the global expression can be obtained merely by replacing the usual derivatives by $\mathrm{D}$ in (12).

Remark 3. A similar result holds for the horizontal Lagrange-Poincaré equations considered in $[1,2]$. One of the goals of these references is to analyse the intrinsic meaning of motion equations for constrained systems with symmetries. Let us recall that, in such a system, the Lagrangian $L$ and the constraints remain invariant under the lifting to $T Q$ of a suitable action of a Lie group $G$ on $Q$. A connection $A$, related to the constraints, is introduced on the principal bundle $Q \stackrel{\pi}{\rightarrow} Q / G$. If $\tilde{\mathfrak{g}}$ is the adjoint bundle to the principal bundle $Q$, the connection $A$ yields an isomorphism $\alpha$ between $T Q / G$ and the Whitney sum $T(Q / G) \oplus \tilde{\mathfrak{g}}$ in the following way

$$
\alpha_{A}[q, \dot{q}]_{G}=(x, \dot{x}, \tilde{v})=\pi_{*}(q, \dot{q}) \oplus[q, A(q, \dot{q})]_{G}
$$


Now, one can define the reduced Lagrangian $\ell: T(Q / G) \oplus \tilde{\mathfrak{g}} \rightarrow \mathbb{R}$ as

$$
\ell(x, \dot{x}, \tilde{v})=L(q, \dot{q}) .
$$

A variation $\delta q$ of a curve in $Q$ is said to be horizontal if $A(\delta q)=0$. In this case, the corresponding variation $\alpha(\delta q(t))$ of the curve $\alpha(q(t))$ in $T(Q / G) \oplus \tilde{\mathfrak{g}}$ is [1]

$$
\alpha(\delta q(t))=\delta x \oplus \tilde{B}(\delta x, \dot{x})
$$

where $\tilde{B}$ is the $\tilde{\mathfrak{g}}$-valued two-form on $Q / G$ defined by

$$
\tilde{B}\left([q]_{G}\right)(X, Y)=\left[q, B\left(X^{h}(q), Y^{h}(q)\right)\right]_{G}
$$

with $X^{h}, Y^{h}$ the horizontal lifts to $Q$ of $X$ and $Y$, and $B$ the curvature of the connection $A$.

The horizontal Lagrange-Poincaré equations for $L$ are defined as the Euler-Lagrange ones for $\ell$ restricted to horizontal variations $\delta q$. A coordinate-free version of them can be written down by introducing an arbitrary covariant derivative D on $T(Q / G)$ and using the covariant derivative $\tilde{\mathrm{D}}$ induced by $A$ on $\tilde{\mathfrak{g}}$.

Under the implicit assumption that the torsion of D vanishes, it is shown in $[1,2]$ that, for horizontal variations $\delta q$,

$$
\delta \int_{t_{0}}^{t_{1}} L(q(t), \dot{q}(t)) \mathrm{d} t=0
$$

if and only if the following horizontal Lagrange-Poincaré equations hold

$$
\frac{\mathrm{D}}{\mathrm{D} t}\left(\frac{\partial \ell}{\partial \dot{x}}\right)(x, \dot{x}, \tilde{v})-\frac{\mathrm{D} \ell}{\mathrm{D} x}(x, \dot{x}, \tilde{v})=-\left\langle\frac{\partial \ell}{\partial \tilde{v}}, \tilde{B}(x)(\dot{x}, .)\right\rangle .
$$

It is easy to see that, for an arbitrary covariant derivative D on $T(Q / G)$, its torsion tensor $T$ must be taken into account in the previous equations. Arguing as above one gets

$$
\frac{\mathrm{D}}{\mathrm{D} t}\left(\frac{\partial \ell}{\partial \dot{x}}\right)(x, \dot{x}, \tilde{v})-\frac{\mathrm{D} \ell}{\mathrm{D} x}(x, \dot{x}, \tilde{v})=-\left\langle\frac{\partial \ell}{\partial \tilde{v}}, \tilde{B}(x)(\dot{x}, .)\right\rangle+T(\dot{q}, .) .
$$

Assuming as in $[1,2]$ the torsion-free requirement for $\mathrm{D}$, the last term clearly vanishes and we recover the horizontal Lagrange-Poincaré equations found in those references.

Again, in any coordinate patch, the expression of horizontal Lagrange-Poincaré equations is independent of the choice of the covariant derivative D.

Remark 4. When considered as a map of the second-order tangent bundle $T(T Q)$ to $T^{*} Q$, the Euler-Lagrange operator turns out to be intrinsic without any choice of connection ( e.g., [9]). The need for connections appears if one prefers to stay in the framework of tangent bundles, as it is usually done, and not to deal with second-order ones.

\section{Acknowledgment}

This work was partially supported by CONICET, Argentina.

\section{References}

[1] Cendra H, Marsden J E and Ratiu T S 2001 Lagrangian reduction by stages Mem. Am. Math. Soc. 722152

[2] Cendra H, Marsden J E and Ratiu T S 2001 Geometric mechanics. Lagrangian reduction, and nonholonomic system Mathematics Unlimited-2001 and Beyond ed B Engquist and W Schmid (Berlin: Springer) pp 221-73

[3] Vershik A M and Faddeev L D 1981 Sel. Math. Sov. 1339 
[4] Vershik A M and Ya Gershkovich V 1994 Non-holonomic Riemannian manifolds Dynamical Systems 7 (Encyclopaedia of Mathematics, vol 16) (Berlin: Springer)

[5] Hehl F W, von der Heyde P and Kerlick G D 1976 Rev. Mod. Phys. 48393

[6] Polchinski J 1998 String Theory vol 2 (Cambridge: Cambridge University Press)

[7] Abraham R and Marsden J E 1967 Foundations of Mechanics (New York: Benjamin)

[8] Kobayashi S and Nomizu K 1963 Foundations of Differential Geometry vol 1 (New York: Wiley)

[9] Marsden J E and Ratiu T S 1994 Introduction to Mechanics and Symmetry (New York: Springer) 